\title{
Les registres médicaux: un outil majeur d'assurance-qualité en médecine
}

\begin{abstract}
Contexte
Un registre médical est un recueil systématique de données médicales et/ou d'économie de la santé homogènes sur un collectif de recherche déterminé. Les possibilités d'utilisation des registres sont nombreuses (par ex. recherche clinique et épidémiologique, recherche sur les soins et les causes des maladies, assurance-qualité, politique de la santé). Les registres offrent également une représentation de la réalité proche de la pratique. La création et la tenue de registres demandent généralement des investissements importants en termes de temps et de moyens financiers, et la plupart des problèmes sont dus à la sous-évaluation de défis liés à la méthode, au manque de participation et à un financement insuffisant. Le manque de ressources destinées aux mesures d'assurance-qualité peut entraîner une perte de la qualité des données et compromettre l'utilité des registres.
\end{abstract}

\section{L'avis de la FMH}

Des registres médicaux de qualité élevée sont essentiels pour évaluer la qualité des traitements et les structures de soins et pour piloter la politique de la santé. C'est pourquoi il convient d'accorder une attention particulière aux points suivants:

\section{Promouvoir la qualité des registres}

Des données valables et complètes garantissent l'utilité scientifique des registres. La méthode utilisée devra respecter les directives internationales en vi- gueur, et ce également afin de garantir la qualité des données. Ces directives recommandent notamment de définir les procédures et la saisie des données au moyen d'un protocole et de saisir des données structurées sur la base de classifications/nomenclatures internationales. De plus, il est essentiel de contrôler les données lors de leur saisie puis à intervalles réguliers (comparaison des données, analyses de plausibilité, audits externes, etc.) et de former les personnes impliquées afin de garantir la qualité de ces données.

Il existe de nombreux critères permettant d'évaluer la qualité et l'utilité d'un registre. Lors de la conception d'un registre, il est indispensable d'avoir un objectif clair et une problématique pertinente. Il est en outre important d'assurer son financement à long terme, de le concevoir de manière transparente et de rendre publics les rapports établis.

Une préoccupation importante de la FMH est de prévenir toute utilisation abusive des données (obligation de confidentialité, aucune possibilité d'identifier les personnes, serveurs sécurisés, locaux fermés, accès aux données réservé aux personnes autorisées, etc.) en tout temps et pour tout type de registres.

\section{Créer des conditions-cadres favorables}

Le succès à long terme d'un registre n'est possible que si les personnes impliquées reconnaissent l'utilité et l'importance de ce dernier. Un autre facteur important est une saisie des données conviviale (conception simple, peu de variables, etc.) et proche de la chaîne de traitement en termes d'emplacement
Correspondance: FMH/DDQ Elfenstrasse 18 CH-3000 Berne 15 Tél. 0313591111 Fax 0313591112 ddq[at]fmh.ch
- Les registres médicaux constituent une importante source de données pour la recherche clinique et épidémiologique, la recherche sur les soins et les causes des maladies, l'assurance-qualité et la politique de la santé. Leur utilité dépend toutefois fortement de la manière dont ils ont été conçus et de la qualité des données qui y figurent.

- Pour que les registres puissent être utilisés de manière optimale, il convient de les concevoir de manière méthodique, de respecter des normes et des critères de qualité précis (contrôle de la qualité des données, outils conviviaux pour la saisie des données, formation des personnes impliquées, notamment celles en charge de la saisie des données), de disposer de concepts en matière de sécurité et de protection des données et d'établir des rapports transparents.

- Les responsables des registres doivent être davantage conscients de l'importance de ressources suffisantes en termes d'argent et de personnel.

- Sur le plan politique, le débat doit porter sur les avantages et les risques des registres et sur les éventuelles adaptations nécessaires des bases légales y relatives. II importe de définir avec tous les partenaires impliqués comment les registres devront être conçus et gérés à l'avenir en Suisse, et ce dans le respect de la protection des données. 
et de temps. Afin de motiver les personnes impliquées, la FMH estime qu'il est primordial que des ressources suffisantes soient allouées à la gestion des registres.

\section{Discuter de la conception et de la gestion des registres sur le plan politique}

Les registres médicaux gagnent de plus en plus en importance en Suisse où de nouveaux registres sont créés en permanence. Sur le plan politique, il serait judicieux de discuter des exigences fondamentales liées à la mise sur pied et à la gestion des registres en y associant les personnes concernées. Les registres doivent par exemple être conçus de manière à ce que les médecins impliqués puissent recevoir un retour d'information (au sens d'un système évolutif) concernant les données qu'ils saisissent, afin de pouvoir en améliorer la qualité si nécessaire. De plus, il est indispensable de saisir les données uniquement dans un but précis et de prendre toutes les mesures nécessaires (par ex. anonymisation ou pseudonymisation lors de la transmission des données) pour éviter les abus. La FMH est disposée à engager la discussion et à rechercher des solutions communes, y compris en ce qui concerne les adaptations légales. 